\title{
Microsurgery: Global Perspectives, An Update
}

Editors

JIN BO TANG

MICHEL SAINT-CYR

CLINICS IN

PLASTIC SURGERY

www.plasticsurgery.theclinics.com

October 2020 • Volume 47 • Number 4 